\title{
Transform Based Image Denoising
}

\author{
${ }^{1}$ K. Sumanth, ${ }^{2}$ CH. Hima Bindu, ${ }^{3}$ T. Srinivasa Rao, ${ }^{4}$ Vijayarangan R. \\ ${ }^{1,2}$ Department of ECE, QIS College of Engineering \& Technology (Autonomous), Ongole, Prakasam district, A.P, INDIA. \\ ${ }^{3}$ Assistant Professor, Department of EIE, Bapatla Engineering College, Bapatla \\ 4. Adviser- Research \& collaborations, QIS College of Engineering \& Technology (Autonomous), \\ Ongole, Prakasam district, A.P, INDIA
}

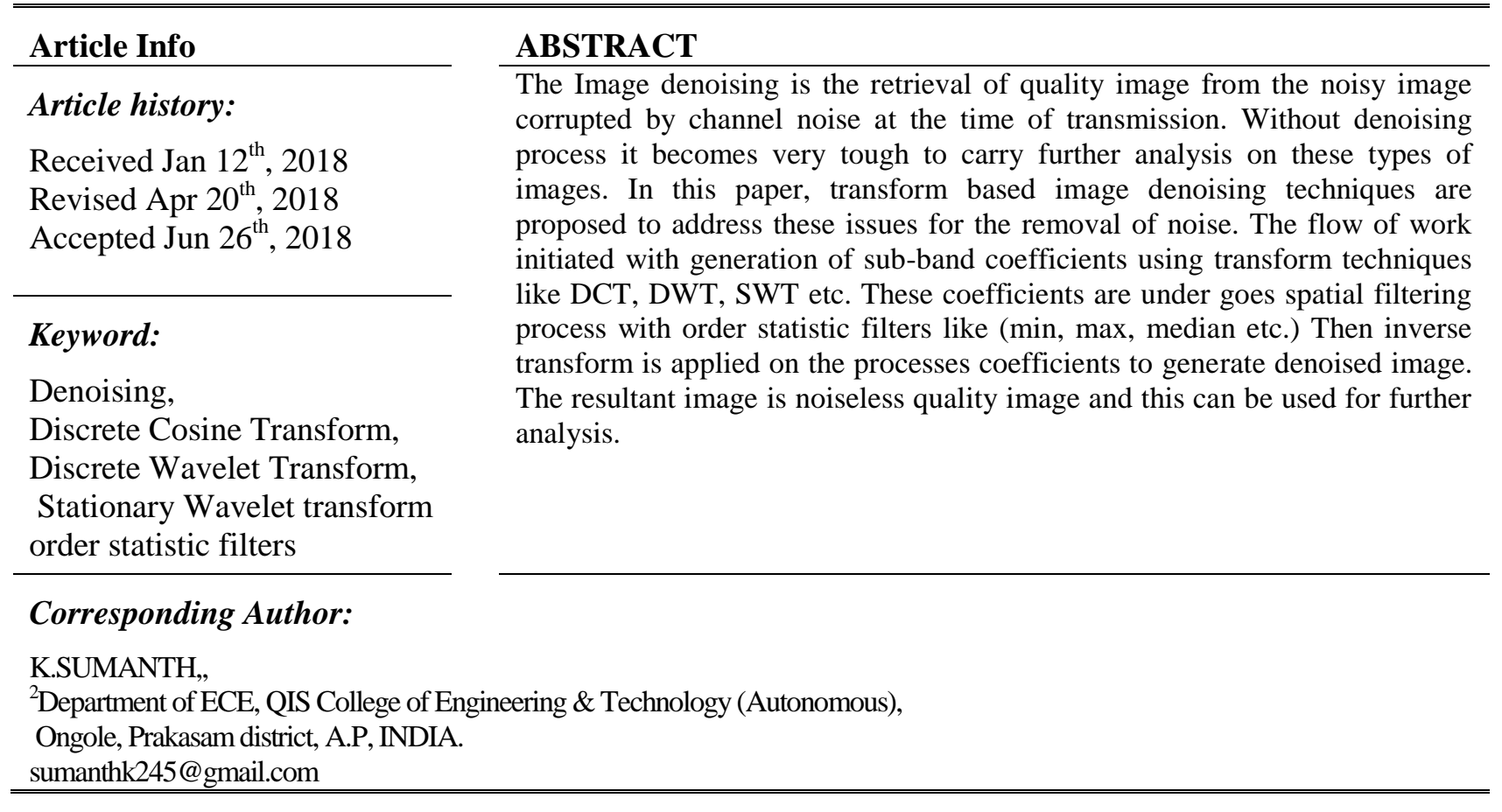

\section{Introduction}

The way of reconstructing an original image from the noisy image is called the image denoising. So to reconstruct the original image we need to remove the unwanted components from the noisy image [11]. And the other way of estimating the original image from the noisy image is referred as image denoising. While removing the original image data from the noisy data we must ensure that we need to preserve the useful information of the image data [5]. To further analyze the image the first step for processing the image is denoising.

Image denoising process to improve the quality of image every algorithms has some filtering and threshold parameters. Image denoising is necessary to improve the quality of image $[1,12]$. The efficient image denoising methods is still a valid challenge at statistics and functional analysis wavelet algorithms are most useful tool for signal processing such as image compression and denoising multiple wavelets are considered for extension of scalar wavelets [2]. To remove noise from original image still exists over past two decades different types noise reduction techniques have been developed [3] it reviews the transform based denoising techniques and performs comparative study and put results for different approaches. Image 
denoising involves the manipulate data of image to produce high quality image [4] it reviews the noise models and classification of image denoising techniques.

\section{Preliminaries}

\subsection{Discrete Wavelet transforms}

Discrete wavelet transform is an analytical tool for analyzing the signals which are occurs in the real time i.e., all the real time signals. Basically the discrete Wavelet transform decomposes the input signal into different basis functions which are also called as the wavelet, a wavelet is a wave like signal which is having the finite duration. Here initial basis function is called as a mother wavelet and the other wavelets are obtained by shifting and dilating the signal. The DWT actually a filtering method which filters the input signal into two frequency components those are high frequency components and low frequency components [6-10]. The basic DWT decomposition process is shown in fig (1) [13]:

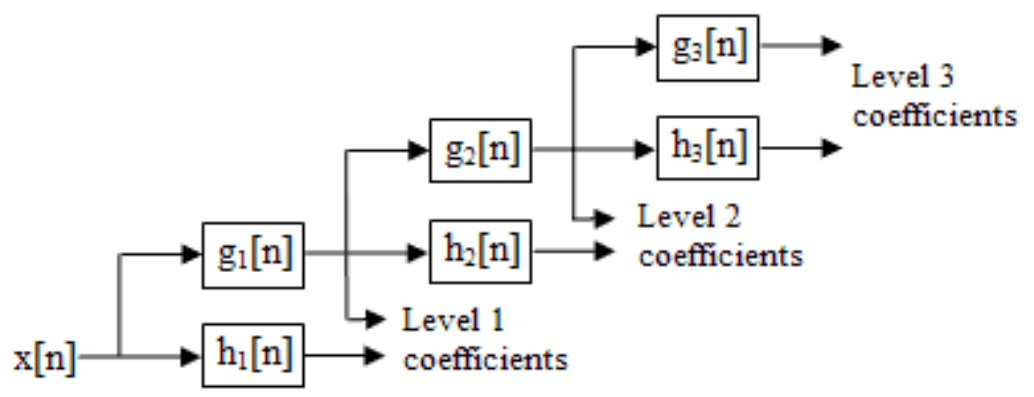

Fig1: DWT decomposition process

As represented in the above the input signal $x(n)$ is decomposed into two parts i.e., $g_{1}(n)$ and $h_{1}(n)$, where the $\mathrm{g}_{1}(\mathrm{n})$ is the high frequency component in the signal $\mathrm{x}(\mathrm{n})$ and $\mathrm{h}_{1}(\mathrm{n})$ is the low frequency component in the signal $\mathrm{x}(\mathrm{n})$ this two terms are called as the level1 coefficients and again these two components will be decomposed i.e., $g_{1}(n)$ is decomposed into $g_{2}(n)$ and $h_{2}(n)$ these two called as the level 2 coefficients and likewise the input signal is decomposed into different levels, but the level of decomposition depends the signal length and sampling frequency $[8,14]$. So we need reconstruct the original signal by using all the coefficients.

\subsection{Discrete Cosine Transforms}

A separate cosine transformation is the last sequence of points in terms of the sum of the differing cone-dependent cosine functions. DCTs are important for many practices in science and engineering [15]. Using cosine as a necessary function is essential for compression because it shows that less cosine needs to be in the same way as conventional ones. While equation differences, cosine shows the specific options of boundary conditions. In particular, the DCT is a Fourier-based shift similar to the Fourier transform apart, but uses only real numbers. DCTs are usually associated with the Fourier series frequency matrix [16]. 


\subsection{Stationary Wavelet Transform}

DWT is a different time shift. The way to restore the variability of this translation is to have on a slightly different dwt mean, the United Nations has destroyed the dwt, to determine the changed stationary wavelet (SWT), it does this by removing steps The sample cost algorithm menu and the replacement of the filter up the prototype by entering the coefficients of the zeros filter. The filter algorithm is a sample called trous, which means "with a socket." On behalf of the detsimiziraniya algorithm filter, first apply to the line and then to the column. The case, however,, although formed four, have the same size image as the original image. Thus, the image of the non-destructive algorithm is shown. The degree of resolution with the cuboid size resolution becomes even more difficult and the size remains the same. [17] The wavelet transformer (SWT) is similar to the separate wavelet exchange (dwt), but only the declining process, which is suppressed by the model, means that SWT is unchanged. The project of the decomposition of SWT 2-D shows in this figure. The stationary wavelet-D, which is changed by 2 (SWT), is based on the idea of a lack of damage. It is applied to a separate wavelet (dwt) and skips both samples forward and up to change the opposite pattern. More precisely, it uses a conversion for each point of the image and saves the proportion of components and uses the lower frequency of each level [18].

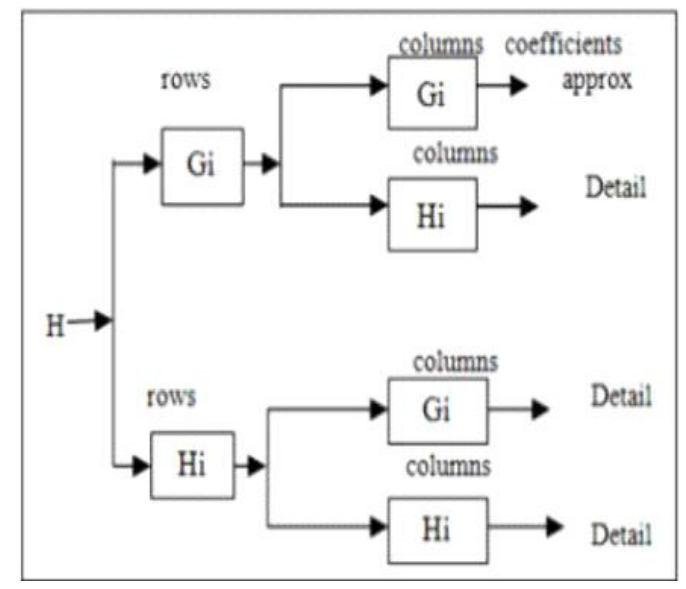

Fig2: SWT decomposition method

\section{Proposed Method}

In this proposed method transform based denoising techniques are introduced to remove the noise on images the flow of proposed method is shown in fig (1). the process of proposed method is given below

Step1: consider the noise image (salt and pepper ) $\mathrm{I}_{\mathrm{N}}(\mathrm{x}, \mathrm{y})$ with a concentration of (0to1).

Step2: Apply transform techniques DCT,DWT \& SWT on the noise images:

$$
\begin{aligned}
& {[\mathrm{S} 1, \mathrm{~S} 2, \mathrm{~S} 3, \mathrm{~S} 4]=\mathrm{DWT}_{2}\left(\mathrm{I}_{\mathrm{N}}(\mathrm{x}, \mathrm{y})\right) \text {--------- (1) }} \\
& {[\mathrm{S} 1, \mathrm{~S} 2, \mathrm{~S} 3, \mathrm{~S} 4]=\mathrm{SWT}_{2}\left(\mathrm{I}_{\mathrm{N}}(\mathrm{x}, \mathrm{y})\right)-----------(2)} \\
& {[\mathrm{S} 1, \mathrm{~S} 2, \mathrm{~S} 3, \mathrm{~S} 4]=\mathrm{DCT}_{2}\left(\mathrm{I}_{\mathrm{N}}(\mathrm{x}, \mathrm{y})\right)---------(3)}
\end{aligned}
$$

Step3: Apply order statistic filter (max/min/median) on above sub bands ( $1, \mathrm{~S} 2, \mathrm{~S} 3, \mathrm{~S} 4)$ respectively.

$\left.\mathrm{S}_{\mathrm{n}=\operatorname{par}}^{1} \mathrm{~S}_{\mathrm{n}}\right\}$

where $\mathrm{n}=1,2,3,4$

par ----->max/min/median 
Step4: Apply inverse transform (IDWT, ISWT, IDCT) on $\mathrm{S}_{\mathrm{n}}^{1}$ where, $\mathrm{n}=1,2,3,4$ sub-bands

$\mathrm{D}(\mathrm{x}, \mathrm{y})=\operatorname{IDWT}_{2}\left(\mathrm{~S}_{1}{ }^{1}, \mathrm{~S}_{2}{ }^{1}, \mathrm{~S}_{3}{ }^{1}, \mathrm{~S}_{4}{ }^{1}\right)(\mathrm{OR})$

$\mathrm{D}(\mathrm{x}, \mathrm{y})=\operatorname{ISWT}_{2}\left(\mathrm{~S}_{1}{ }^{1}, \mathrm{~S}_{2}{ }^{1}, \mathrm{~S}_{3}{ }^{1}, \mathrm{~S}_{4}{ }^{1}\right)(\mathrm{OR})$

$\mathrm{D}(\mathrm{x}, \mathrm{y})=\operatorname{IDCT}_{2}\left(\mathrm{~S}_{1}{ }^{1}, \mathrm{~S}_{2}{ }^{1}, \mathrm{~S}_{3}{ }^{1}, \mathrm{~S}_{4}{ }^{1}\right)$

Step5: The image $\mathrm{D}(\mathrm{x}, \mathrm{y})$ is the denoised image by removing salt and pepper noise.

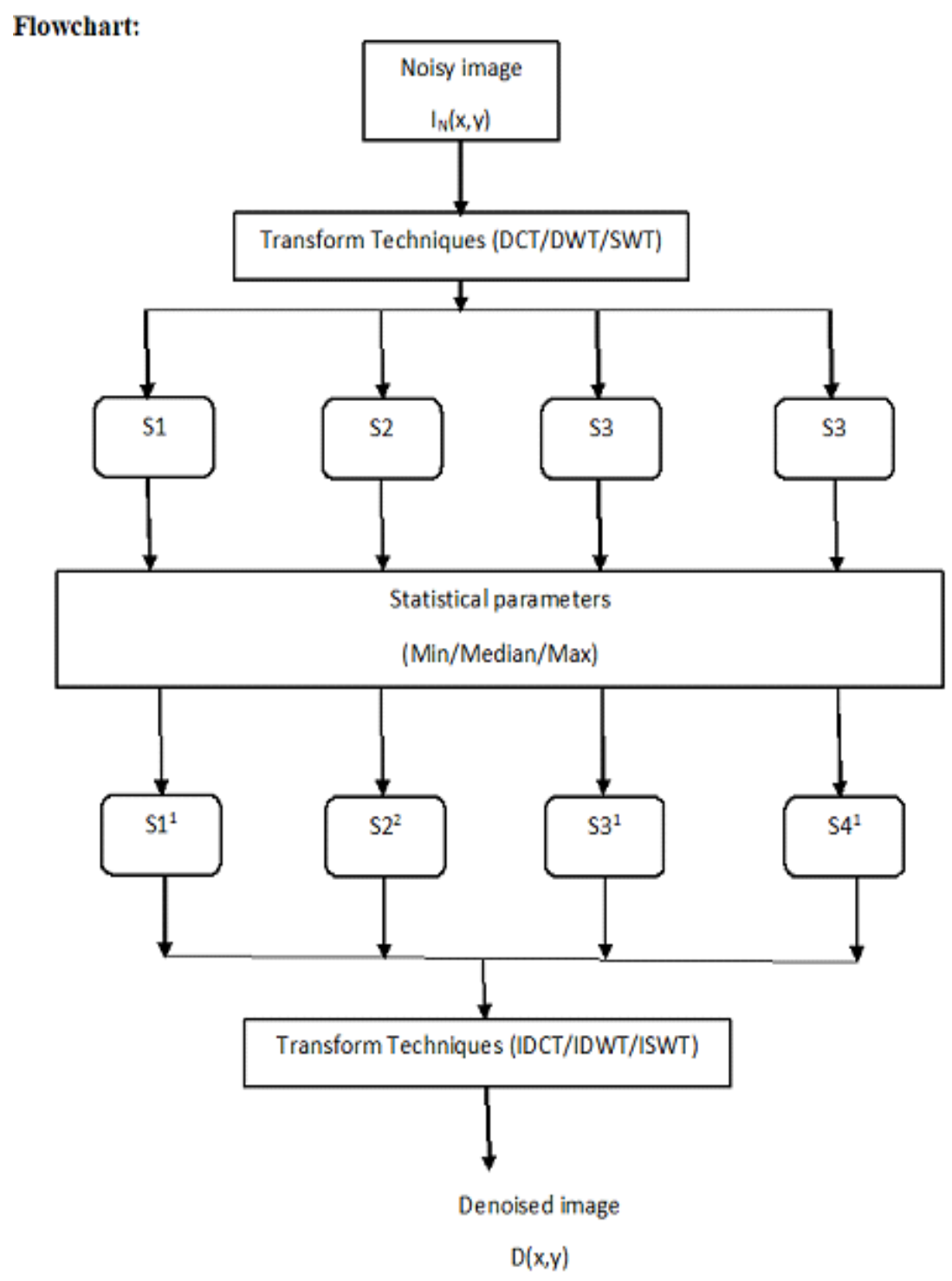

\section{Experimental Results}

In this paper the proposed work is verified on various noised images with Matlab platform the basic functions of transform techniques are haar' the statistical parameters are performed with $3 \times 3$ mask size the resultant images shown in the figures. The resultant image consist more noiseless information for further analysis. The output of the DCT for gray scaled image is shown as shown as follows using min, median and max filters is shown as follows. The results images of DCT, SWT \& DWT are shown in the following figures from Fig 4-9 on two types of images respectively. From these figures we can analyze visually the DWT is producing better results with median filter instead of all other transforms and other order statistic filters. 


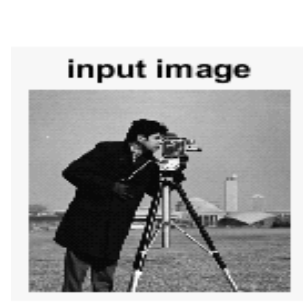

output of $\min$ filter
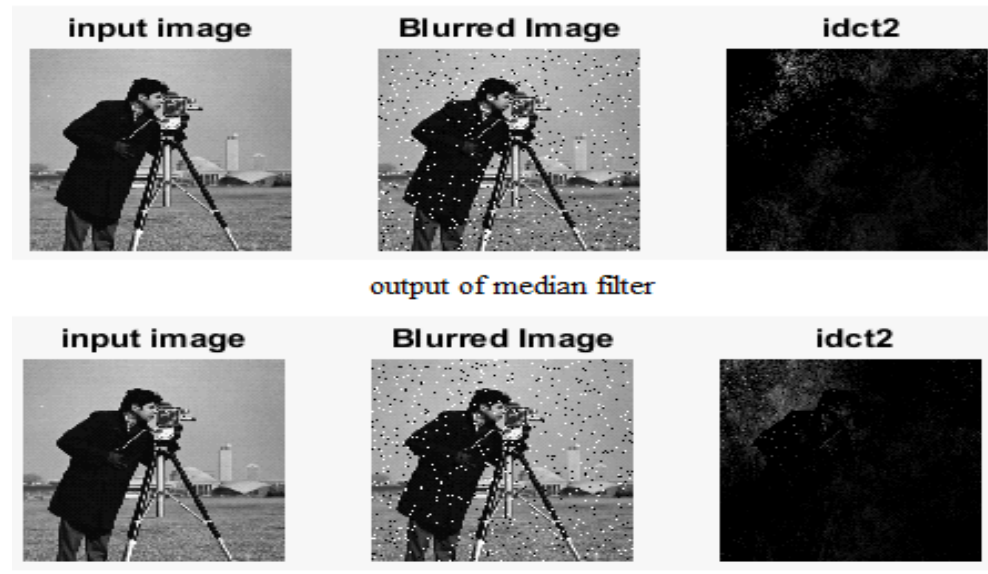

output of median filter

Blurred Image
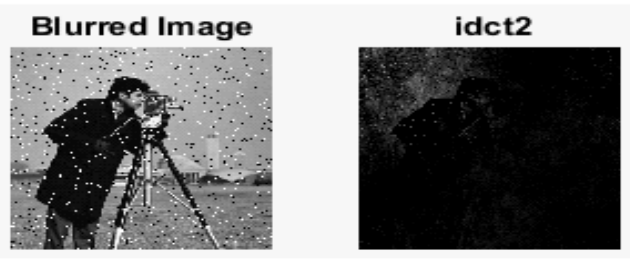

output of max filter
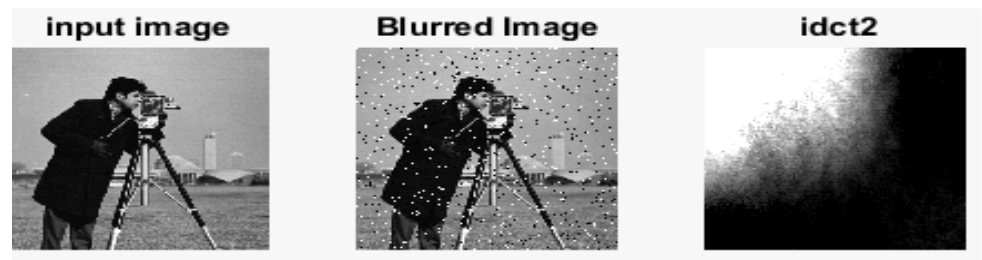

Fig 4: Denoised image with DCT using the min, median, max filters

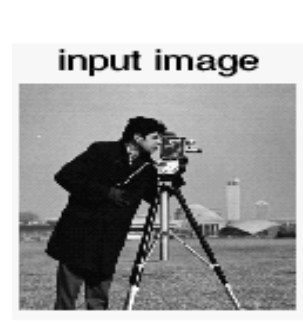

output of median filter
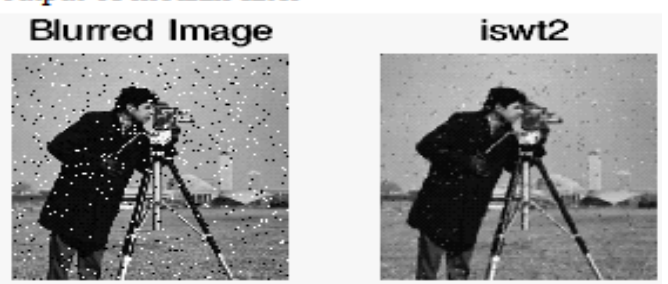

output of min filter
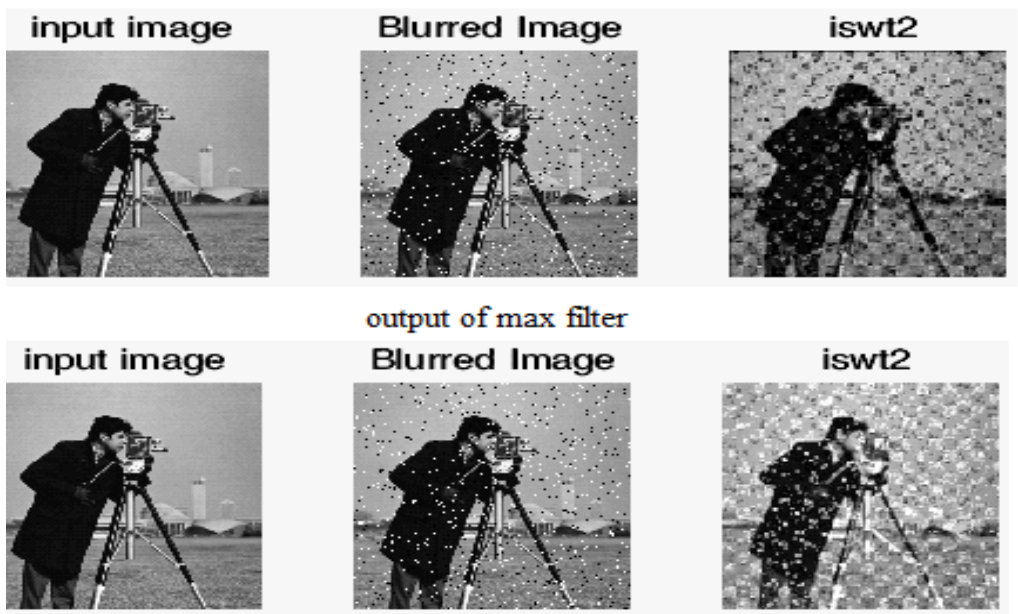

output of $\max$ filter
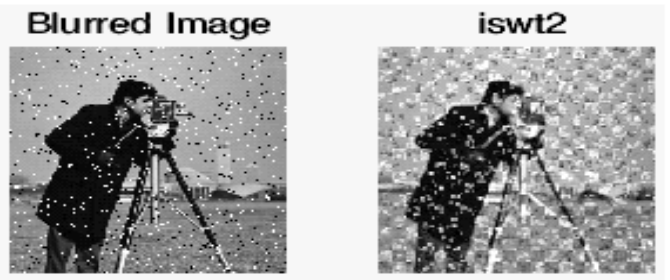

Fig 5: Denoised image with SWT using the min, median, max filters 


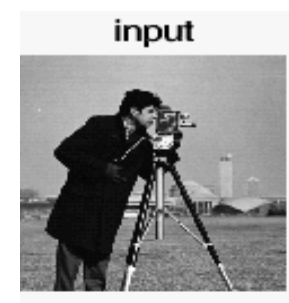

output of $\mathrm{min}$ filter
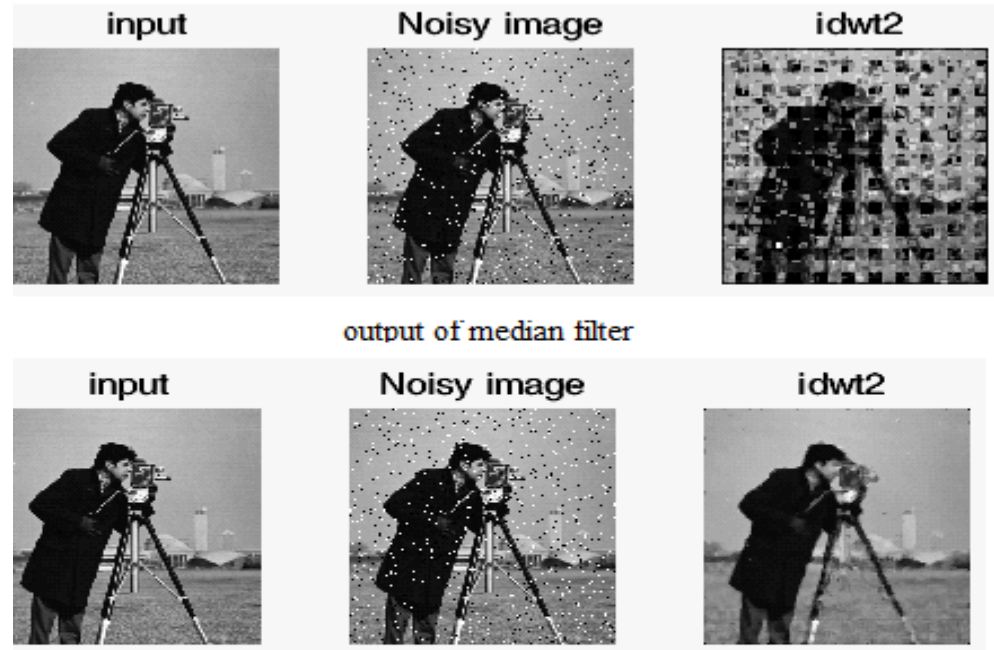

output of median tilter
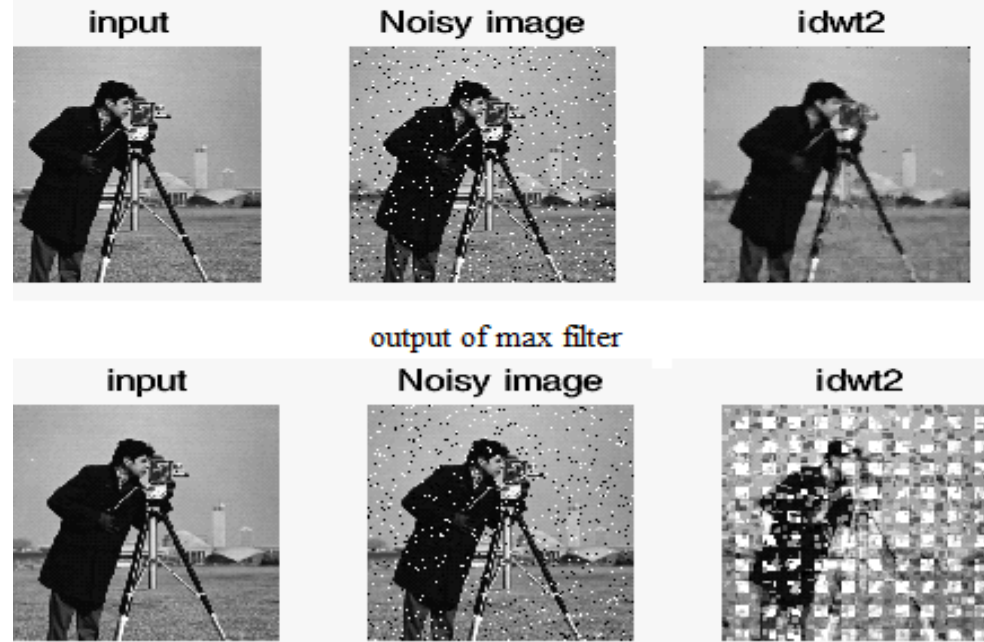

output of max filter Noisy image
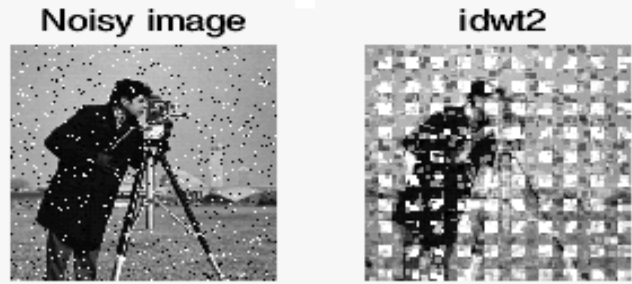

Fig 6: Denoised image with DWT using the min, median, max filters

output of $\min$ filter
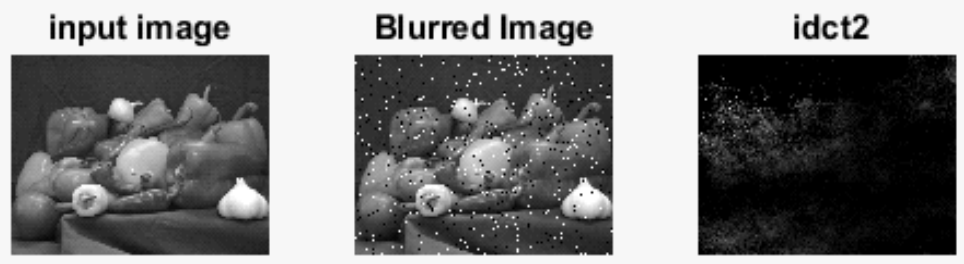

output of median filter
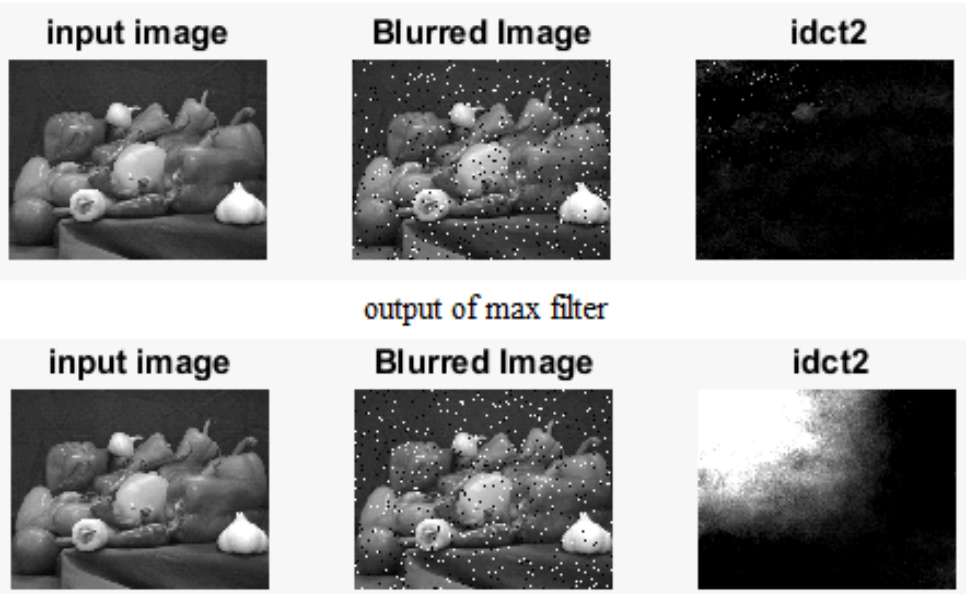

output of max filter
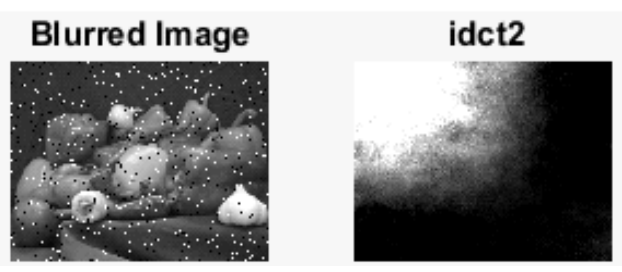

Fig 7: Denoised image with DCT using the min, median, max filters 


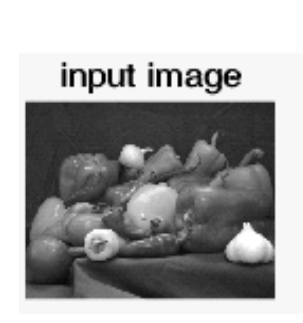

output of $\mathrm{min}$ filter
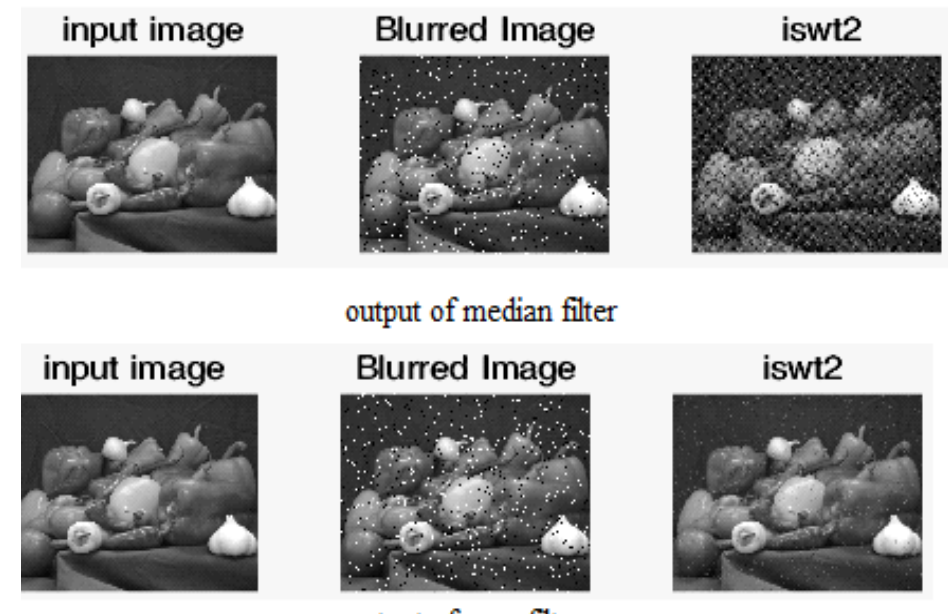

output of median filter
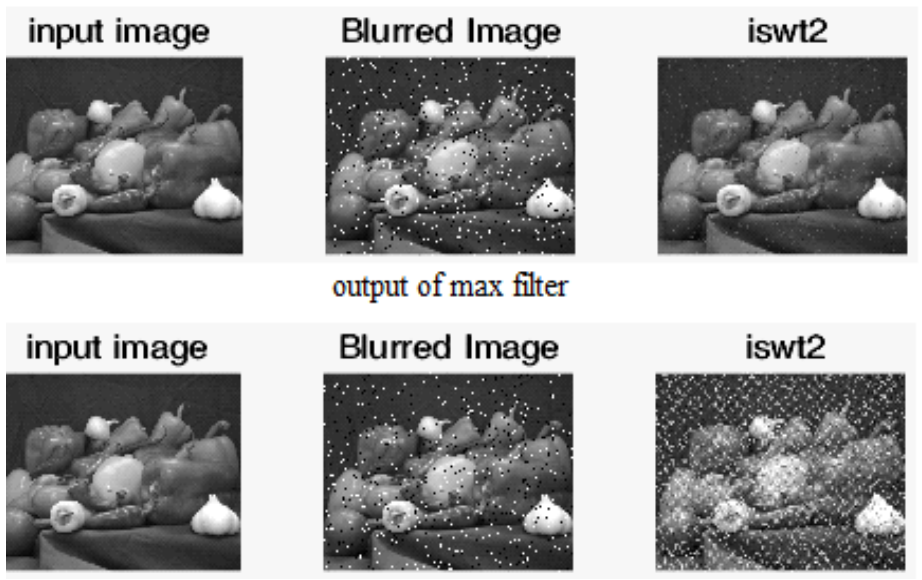

output of max filter
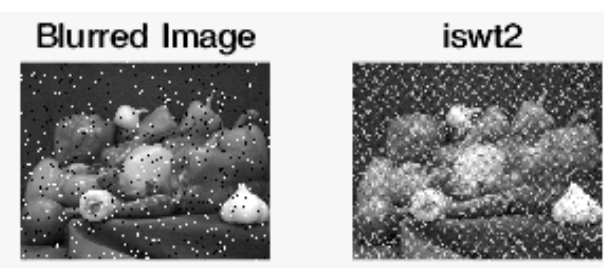

Fig 8: Denoised image with SWT using the min, median, max filters output of min filter
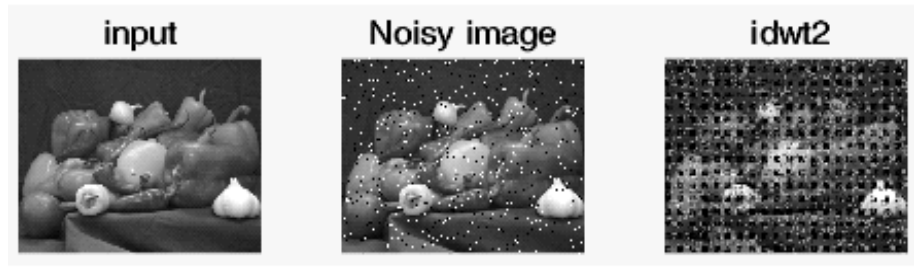

output of median filter
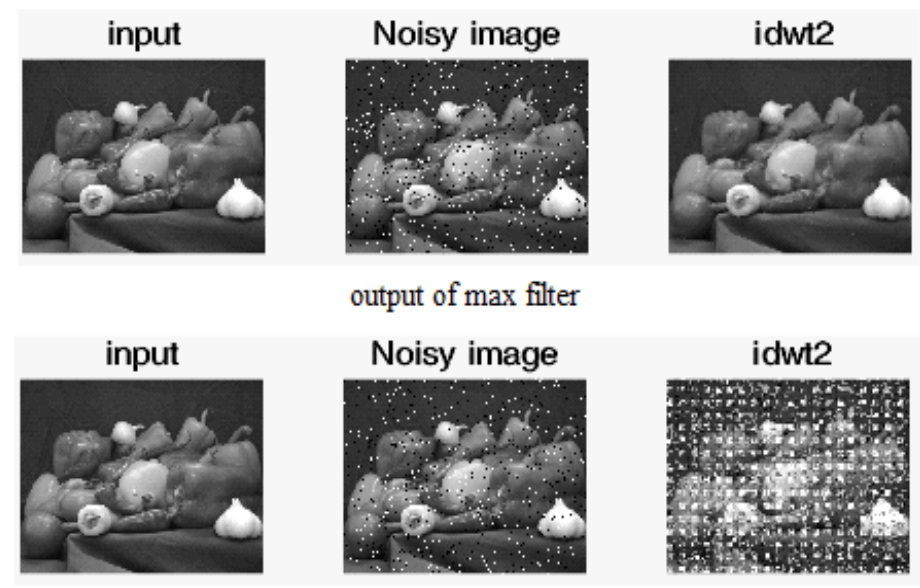

output of max filter
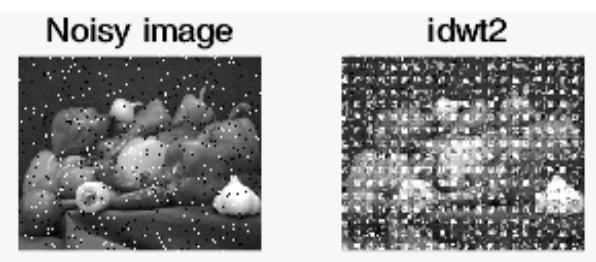

Fig 9: Denoised image with DWT using the min, median, max filters

\section{Conclusion}

The proposed transform based denoising image technique is verified on salt and pepper noisy image with concentration of (0to1). In this work 3 transform techniques are used (DWT, SWT and DCT) for the generation of sub-band coefficients the spatial filtering is verified with $3 \times 3$ order statistical filters for more 
fine details on sub-band coefficients the final resultant image is noise less. This work can be extendable with various transform techniques and various spatial filtering approaches. Out of all these DWT is giving better results with median filter. This work can be extendable with various transforms and various spatial filtering techniques.

\section{References}

1. yali Liu ,2015. "Image denoising method based on threshold, wavelet transform and genetic algorithm" Int. Journal Of image processing and pattern Recognition vol.8,No.2(2015). pp 29-40.

2. Mr.Sachin Ruikar. "wavelet based image denoising technique" international journal of advanced computer science and applications, vol. 2, No.3, March 2011.

3. Nidhi Soni "Transform based image denoising" international conference on recent innovations in signal processing and embedded systems, 2017.

4. jyotsna patil "A Comparative study of Image Denoising Techniques" international journal of innovative research in science, vol.2, Issue 3, March 2013.

5. D. Donoho, "Denoising by soft thresholding", IEEE. Trans. Inf. Theory, vol. 41, no. 3, pp. 613-627, May 1995

6. I. Daubechies, Ten Lectures on Wavelets, PA, Philadelphia:SIAM, 1992.

7. Y. Meyer, Wavelets and Operators, New York:Cambridge Univ. Press, 1992.

8. A. Cohen, I. Daubechies, P. Vial, "Wavelets on the interval and fast wavelet transforms", Appl. Comput. Harmon. Anal., vol. 1, pp. 54-81, 1993.

9. James S. Walker, -Wavelets Based Image Processing, Department of Mathematics University of Wisconsin Eau Claire.

10. S. Grace Chang, Bin Yu and M. Vattereli, Wavelet Thresholding for Multiple Noisy Image Copies, IEEE Trans. Image Processing, vol. 9, pp.1631- 1635, Sept. 2000.

11. L. Yaroslavsky. Digital Picture Processing - An Introduction. Springer Verlag, 1985.

12. A. Buades, B. Coll, and J Morel. On image denoising methods. Technical Report 2004- 15, CMLA, 2004.

13. S. Mallat, "A theory for multiresolution signal decomposition: The wavelet representation," IEEE Trans. Pattern Anal. Machine Intell., vol. 11, pp. 674-693, July 1989. 
14. Y. Piao, I. Shin, and H. W. Park, "Image resolution enhancement using inter-subband correlation in wavelet domain,” in Proc. Int. Conf. Image Process., 2007, vol. 1, pp. I-445-44.

15. Discrete Cosine Transform: algorithms, advantages and applications by K.Rammohan rao, P.Yip.

16. Ahmed, N., T. Natarajan, and K. R. Rao. 1974. On image processing and a discrete cosine transform. IEEE Transactions on Computers C-23(1): 90-93.

17. Hasan Demirel And Gholamreza Anbarjafari, 2011. "Image Resolution Enhancement By Using Discrete And stationary Wavelet Decomposition,’'Ieee Transactions On Image Processing, 20: 5.

18. Vijayaarjunan, R., B. Siva Chandra Mahalingam, M. Arun, 2013. "Image Resolution Enhancement Using Multi Resolution Wavelet Transformation". 